http://jmscr.igmpublication.org/home/ ISSN (e)-2347-176x ISSN (p) 2455-0450

crossref DOI: https://dx.doi.org/10.18535/jmscr/v7i10.52

Journal Of Medical Science And Clinical Research

\title{
Incidence of Steroid-Induced Diabetes Mellitus caused by Dexamethasone used for prevention of Chemotherapy induced nausea and vomiting
}

\author{
Authors \\ Sanjay Kumar Thakur ${ }^{1 *}$, Sukhesh Purush Dhakal ${ }^{1}$, Umesh Bogati ${ }^{1}$, Anil Shah ${ }^{2}$, \\ George Jung Bush Katuwal ${ }^{2}$, Suman Supratik ${ }^{3}$, Bishnu Dutta Paudel ${ }^{1}$ \\ ${ }^{1}$ National Academy of Medical Sciences, Bir hospital, Kathmandu, Nepal \\ ${ }^{2}$ Annapurna Research centre, Maitighar, Kathmandu, Nepal \\ ${ }^{3}$ St. Xavier's College, Maitighar, Kathmandu, Nepal \\ *Corresponding Author \\ Sanjay Kumar Thakur \\ National Academy of Medical Sciences, Bir hospital, Kathmandu, Nepal
}

\begin{abstract}
New onset diabetes is often noticed when steroid is used especially in higher dosage and for a prolonged duration. Aim of this study was to estimate proportion of patients' developing new onset diabetes mellitus after receiving dexamethasone for the prevention of Chemotherapy induced nausea and vomiting (CINV).

Methods: A prospective hospital based observational study was conducted from May, 2018 to April 2019 on chemotherapy treated cancer patients of the clinical oncology department of Bir hospital in Kathmandu who were kept on dexamethasone for the prevention of CINV. Inclusion criteria was: Adult cancer patients who were prescribed chemotherapy along with dexamethasone for the prevention of CINV. Exclusion criteria were: Previously diagnosed cases of diabetes mellitus or patients diagnosed of any other similar conditions or patients were using any other drugs (including long term steroid therapy for other conditions) known to cause hyperglycemia/ diabetes mellitus. At 0, 3 or 6 months of follow up patients were tested for blood sugar or HBAlc for the evaluation of onset of diabetes mellitus. In newly diagnosed diabetic cases a cumulative dose and duration of dexamethasone use were calculated and analyzed for their relationships and tested for statistical significance. X2 test or fisher exact test, and tor $z$ tests were applied depending on variable and sample size. Regression analyses was performed to examine the associations.

Result: A total of 47 patients were evaluated for the onset of dexamethasone (used for the prevention of CINV) induced diabetes mellitus during the study period from May, 2018 to April 2019. Out of 47 patients evaluated, we found 6 (12.7\%) patients of new onset diabetes mellitus requiring anti diabetic treatment due to dexamethasone therapy even after withdrawal of dexamethasone. The higher (5/6) number of patients developed diabetes after getting $\geq 3$ days cycles and $\geq 20 \mathrm{mg} /$ day cumulative dose of dexamethasone per cycle. Obese patients developed diabetes mellitus more frequently than non-obese patients. Diabetes mellitus were found more frequently when they were prescribed dexamethasone along with Oxaliplatin, Paclitaxel, CAF, CAV, CAPOX and TAC regimens (Table 2). However, none of the above findings were found statistically significant.

Conclusion: Incidence of dexamethasone induced diabetes mellitus used for prevention of CINV was found to be around $13 \%$ (p-0.81).

Keywords: Dexamethasone, CINV, Diabetes mellitus.
\end{abstract}




\section{Introduction}

The diagnosis of diabetes can be made as per the WHO criteria when Patient complains of symptoms suggesting diabetes and blood sugar test results of Fasting $\geq 7.0(\geq 126)$ or 2 hours after glucose load or a Random blood sugar $\geq 11.1$ $(\geq 200)$ from Venous plasma $\mathrm{mmol} / \mathrm{l}(\mathrm{mg} / \mathrm{dl})$ or a HBA1c value of $>\_6.5$ or more is obtained ${ }^{1}$. There are many known secondary causes and well recognized risk factors for developing secondary diabetes. Both endogenous as well as exogenous factors are responsible for the onset of diabetes. One of the most important and well known risk factors for the DE novo onset of diabetes mellitus is the steroid use. Steroids are prescribed both pre and post chemotherapy administration to prevent CINV. New onset diabetes are often noticed when steroid is used especially in high dose and for prolonged duration. Some of them may require antidiabetic treatment even after removal of steroids.

Based on the emetogenic potential of a drug different antiemetic drugs are prescribed pre and post chemotherapy periods. Most patients are also prescribed low to high dose dexamethasone. An average of 1 day prior to and 3 to 5 days after chemotherapy, dexamethasone is prescribed at each cycle of chemotherapy. Generally more than 6 such chemotherapy cycles are prescribed along with dexamethasone in a patient. So, cumulative dose and duration of dexamethasone is risk for DE novo diabetes mellitus. Our patients on general carry many other risk factors for diabetes such as pre morbid obesity, high BMI, inactivity, high calorie intake, and prediabetic conditions. The fact that steroid induced diabetes is well known but the accurate estimation of steroid induced diabetes in our population needs to be quantified. Moreover, the dose and duration of dexamethasone use can be linked to the onset of diabetes especially among risk patients. By knowing it, we can prescribe lifestyle changes and risk reduction interventions to the patients who are at risk of developing diabetes mellitus prior to chemotherapy requiring dexamethasone. More importantly, we can modify our prescribing habits of dexamethasone dose and duration as minimum as possible for the optimally required dose and duration for the prevention of CINV. The aims of the study were to estimate proportion of patients receiving dexamethasone for the prevention of CINV develop diabetes mellitus and to explore relationship between cumulative dose of dexamethasone use and new onset of Diabetes Mellitus requiring antidiabetic treatments.

\section{Methods}

A prospective hospital based observational study was conducted on chemotherapy treated cancer patients who were kept on dexamethasone for the prevention of CINV. All enrolled non-diabetic Cancer patients in the clinical oncology department of NAMS, Bir hospital who were prescribed dexamethasone along with chemotherapy were followed up during chemotherapy cycles to look for the new onset of hyperglycemia and diabetes mellitus. Baseline and at follow up of 3 or 6 months after chemotherapy plus dexamethasone treatment, symptoms and biochemical evaluation were done to find out new onset diabetes mellitus requiring diabetic treatment. Cumulative dose of dexamethasone intake were calculated at the end of the chemotherapy regimen or at the onset of diabetes mellitus. Enrollment started from May, 2018 to April 2019. Subject enrollments were done after taking ethical approval from the IRB, informed consent based on the set inclusion and exclusion criteria. Inclusion criteria was: Adult cancer patients enrolled in department of clinical oncology at Bir hospital and who were prescribed chemotherapy along with dexamethasone for the prevention of CINV. The exclusion criteria were: Previously diagnosed cases of diabetes, any other conditions or drugs (including long term steroid therapy for other conditions) known to cause hyperglycemia/ diabetes mellitus.

Procedures and protocols: A venous blood sample was sent for testing of blood sugar at 0,3 or at 6 months follow up(Testing blood sugar at 6 
month follow up was avoided when 3 month's sugar value came normal). For Fasting blood sugar (FBS) testing-After an 8 hours of fasting, a venous blood sample was drawn and tested for sugar level. Post prandial blood sugar testing was done-after 2 hours of meal. Random venous blood sample tested for random sugar level or for HBA1c .Those prescribed by WHO protocol, techniques and procedures for the diagnosis of diabetes mellitus were followed. All baseline characteristics including demography, comorbidities, drug use were analyzed before enrollment into the study. Biochemistry including RFT, LFT, Electrolytes, and CBCwere tested as per the departmental protocol for chemotherapy at the point of enrollment. At 0, 3 and/or $6(6$ month testing was optional when 3 month blood sugar was normal) months of follow up blood sugar or HBA1c were tested for the evaluation of late onset of diabetes mellitus. In newly diagnosed diabetic cases a cumulative dose and duration of dexamethasone use were calculated, analyzed for their relationships and tested for statistical significance.

\section{Statistical Analysis}

Data were categorized in categorical and noncategorical. Qualitative data were tested by using X2 test or fisher exact test. Quantitative data were tested using $\mathrm{t}$ or $\mathrm{z}$ tests depending on sample size. Mean values were compared using the test (two categories) and one-way analysis of variance (more than two categories). Proportions were compared using two-way tables and chi square tests. Univariate and multivariate regression analyses were performed to examine the association between the incidence of diabetes and clinical variables. Logistic regression analysis was applied for analysis of high dexamethasone usage which was defined as a cumulative dose of dexamethasone $>156 \mathrm{mg}$, which is equivalent to six cycles of moderately emetogenic chemotherapy that included dexamethasone as an antiemetic. $\mathrm{P}<0.05$ was considered statistically significant. Statistical analyses were performed using SPSS ver. 17.0.

\section{Results}

Total 47 patients were evaluated for the onset of dexamethasone (used for the prevention of CINV) induced diabetes mellitus during the study period from May, 2018 to April 2019. Out of 47 patients, we found 6 (13\%), (p-0.81) who developed diabetes mellitus due to dexamethasone therapy. Among those who developed diabetes mellitus, 4 patients were females and 2 were males of age between 40-59 years. The higher (5/6) number of patients developed diabetes after getting $\geq 3$ days cycles and $\geq 20 \mathrm{mg} /$ day cumulative dose of dexamethasone per cycle of chemotherapy. Obese patients develop diabetes mellitus more frequently than non-obese, i.e., obese (3/6), over weight (2/6) and in normal weight (1/6) respectively, developed diabetes mellitus (Table 1). We found patients developing diabetes mellitus more frequently when they were prescribed dexamethasone along with Oxaliplatin, Paclitaxel, CAF, CAV, CAPOX and TAC regimens (Table 2). However none of them were found statistically significant.

\section{Tables:}

\section{A. Dose/duration of dexamethasone therapy and newly diagnosed DM}

\begin{tabular}{|c|c|c|c|c|}
\hline Dose per cycle & Mild to Moc & $<20 \mathrm{mg} /$ day $)$ & High do & mg/day) \\
\hline Duration per cycle & $<3$ days & $>3$ days & $<3$ days & $>3$ days \\
\hline No. of Diabetics & 0 & 1 & 1 & 4 \\
\hline
\end{tabular}


B. Sex, Age and BMI and Cycle, Dose, of dexamethasone use for newly diagnosed DM

\begin{tabular}{|c|c|c|c|c|}
\hline & & Diabetes & Non-Diabetes & p-Value \\
\hline \multirow[t]{2}{*}{ Sex } & Male & 2 & 18 & \multirow[t]{2}{*}{0.62} \\
\hline & Female & 4 & 23 & \\
\hline \multirow{3}{*}{ Age } & $20-30$ & 0 & 4 & \multirow{3}{*}{0.49} \\
\hline & $40-59$ & 6 & $\frac{4}{33}$ & \\
\hline & $60-79$ & 0 & 4 & \\
\hline \multirow{3}{*}{ Cycle } & & & & \multirow{3}{*}{0.75} \\
\hline & $\leq 3$ days & 1 & 5 & \\
\hline & $\geq 3$ days & 5 & 36 & \\
\hline \multirow{2}{*}{ Dose } & $\leq 20 \mathrm{mg}$ days & 1 & 1 & \multirow{2}{*}{0.10} \\
\hline & $\geq 20 \mathrm{mg}$ days & 5 & 40 & \\
\hline & & & & \multirow{4}{*}{0.69} \\
\hline \multirow[t]{3}{*}{ BMI } & Normal & 1 & 3 & \\
\hline & Over Weight & 2 & 12 & \\
\hline & Obese & 3 & 26 & \\
\hline
\end{tabular}

C. Chemotherapy regimen plus dexamethasone and new onset of Diabetes mellitus

\begin{tabular}{|c|c|c|c|}
\hline \multirow{2}{*}{ Drugs } & \multicolumn{2}{|c|}{ Diabetes } & \multirow[t]{2}{*}{ P- Value } \\
\hline & Diabetes & Non-Diabetes & \\
\hline CHOP & 0 & 4 & \multirow{17}{*}{0.81} \\
\hline Oxaliplatin & 1 & 2 & \\
\hline Pacli & 1 & 6 & \\
\hline Epi & 0 & 3 & \\
\hline $\mathrm{CAF}$ & 1 & 4 & \\
\hline BETROXMIDE & 0 & 1 & \\
\hline ECF & 0 & 1 & \\
\hline CAV & 1 & 0 & \\
\hline GemCis & 0 & 3 & \\
\hline ECT & 0 & 1 & \\
\hline CAPOX & 1 & 5 & \\
\hline AC-T regiment & 0 & 1 & \\
\hline DC-T regiment & 0 & 1 & \\
\hline TAC regiment & 1 & 6 & \\
\hline FOLFOX & 0 & 1 & \\
\hline Docetaxel & 0 & 1 & \\
\hline Epi & 0 & 1 & \\
\hline
\end{tabular}

\section{Discussion}

By several antiemetic guidelines, antineoplastic agents are categorized as per the emetogenic risk and matched to specific antiemetic regimens to reduce the degree of CINV; dexamethasone is used in combination with serotonin (5hydroxytryptamine 3 [5-HT3]) or neurokinin-1 (NK-1) receptor antagonists for high or moderate emetogenic risk chemotherapy, or as a single agent when low-emetogenic chemotherapeutic agents are prescribed in both the acute and delayed phases ${ }^{2}$.Keeping in mind the findings of our study which showed no statistical difference in the occurrence of de nevodiabetes mellitus even with the higher dosage of dexamethasone use, our current practice to use the dose and duration of dexamethasone for CINV is an optimal approach and needed no alteration in our current practice. In clinical practice, for an antiemetic effect to achieve, dexamethasone is mostly administered repeatedly over longer periods of time while cancer patients are kept on chemotherapy. Physiologically, it increases the risk of adverse systemic effects as well as diabetes ${ }^{3}$. The hormonal adverse effects of it are adrenal suppression, hyperlipidemia, growth suppression, 
gynecomastia, and amenorrhea. Hyperglycemia is frequently overlooked ${ }^{4-5}$. Glucocorticoids bring about a state of relative insulin resistance. Glucocorticoids down regulate glucose transporter 4 (GLUT 4) in muscle, which requires the high amount of insulin for the glucose uptake by cells causing increased output of glucose by the liver, inhibit insulin binding to the insulin receptor on cells, and a decrease insulin secretion from islet cells ${ }^{6}$. Thus, although found no statistically significant relationship of dexamethasone with development of new onset diabetes mellitus in our study, cases of diabetes were more found in patients with higher dosage and duration of dexamethasone therapy especially in those patients having high BMI. A systematic literature review was conducted using 9 electronic databases. The study revealed that therapies using Glucocorticoids might result in elevated FPG, FINS, and HOMA-IR, and thereby contribute to Insulin Resistance in healthy individuals. Therefore, steroids administration may exacerbate pre-diabetes or undiagnosed diabetes. It can transform mild diabetes into a clinically severe illness, even sometimes leading to a hyperglycemic non-ketotic hyperosmolar coma. Although, the symptoms of hyperglycemia, such as thirst, dry mouth, weakness, weight loss, and polyuria and lethargy, are also common in patients with advanced stage cancer for unrelated reasons such as the tumor itself, certain medicines, metabolic imbalance, and psychological problems symptomatic basis of diagnosing diabetes in these cases are often missed. That's why, the diagnosis of diabetes in patients receiving chemotherapy is most often delayed in the absence of a high degree of clinical suspicion. The principles of early detection and risk factor modification are an important task for both the steroid and non-steroid induced diabetes and insulin resistance ${ }^{7}$. Diagnosis of impaired fasting glucose or impaired glucose tolerance prior to the initiation of chronic glucocorticoids is a recommended approach for the better identification of the case and decision making for those who would benefit from non- steroid treatment. On the whole, in our study observations showed similar findings to a study where steroid dose equivalent to $>12 \mathrm{mg}$ dexamethasone and longer acting steroids caused greater degree of hyperglycemia compared to a dose $<12 \mathrm{mg}^{8}$. This study needed a further large scale study to reach a conclusion and show dexamethasone effect on a larger population to recommend any modification in the current clinical practice of dexamethasone use with chemotherapy for the prevention of CINV in terms of its dose and the duration around chemotherapy cycles.

\section{Conclusion}

Incidence of dexamethasone induced diabetes mellitus used for prevention of CINV was found to be $13 \%(\mathrm{p}-0.81)$.High cumulative dose of dexamethasone and obese patients were important risk factors associated with the new onset of diabetes mellitus requiring diabetic treatment.

\section{Reference}

1. B. M. frier, m. Fisher. Presenting problems in diabetes mellitus, Davidson's principles and practice of medicine. 20th edition. Pages 806 - 808, 817 - 820.

2. NCCN Clinical Practice Guidelines in Oncology: antiemesis. Version 2, 2014 [Internet]. Fort Washington, PA: National Comprehensive Cancer Network; 2014 [cited 2015 Mar 1]. Available from: http://www.nccn.org/professionals/physici an_gls/pdf/antiemesis.pdf.

3. Vardy J, Chiew KS, Galica J, Pond GR, Tannock IF. Side effects associated with the use of dexamethasone for prophylaxis of delayed emesis after moderately emetogenic chemotherapy. Br J Cancer. 2006; 94:1011-5.

4. Fardet L, Kassar A, Cabane J, Flahault A. Corticosteroid induced adverse events in adults: frequency, screening and prevention. Drug Saf. 2007; 30:861-81. 
5. Han HS, Park JC, Park SY, Lee KT, Bae $\mathrm{SB}$, Kim HJ, et al. A prospective multicenter study evaluating secondary adrenal suppression after antiemetic dexamethasone therapy in ccancer patients receiving chemotherapy: a Korean South West Oncology Group Study. Oncologist. 2015; 20:1432-9.

6. Rafacho A, Ortsater H, Nadal A, Quesada I. Glucocorticoid treatment and endocrine pancreas function: implications for glucose homeostasis, insulin resistance and diabetes. J 0. 2014; 223:R49-62.

7. Jessica L. Hwang, Roy E. Weiss. Steroidinduced diabetes: a clinical and molecular approach to understanding and treatment. Diabetes Metab Res Rev. 2014 Feb; 30(2): 96-102.

8. Healy SJ, 55Nagaraja HN, 55Alwan D, 55Dungan KM. Prevalence, predictors, and outcomes of steroid-induced hyperglycemia in hospitalized patients with hematologic malignancies. Endocrine. 2017 Apr; 56(1):90-97. doi: 10.1007/s12020-016-1220-2. Epub 2017 Jan 6. 